\title{
A Hopf-power Markov chain on compositions
}

\author{
C.Y. Amy Pang \\ Department of Mathematics, 450 Serra Mall, Stanford University, CA 94305, USA
}

\begin{abstract}
In a recent paper, Diaconis, Ram and I constructed Markov chains using the coproduct-then-product map of a combinatorial Hopf algebra. We presented an algorithm for diagonalising a large class of these "Hopf-power chains", including the Gilbert-Shannon-Reeds model of riffle-shuffling of a deck of cards and a rock-breaking model.

A very restrictive condition from that paper is removed in my thesis, and this extended abstract focuses on one application of the improved theory. Here, I use a new technique of lumping Hopf-power chains to show that the Hopf-power chain on the algebra of quasisymmetric functions is the induced chain on descent sets under riffleshuffling. Moreover, I relate its right and left eigenfunctions to Garsia-Reutenauer idempotents and ribbon characters respectively, from which I recover an analogous result of Diaconis and Fulman (2012) concerning the number of descents under riffle-shuffling.

Résumé. Dans un récent article avec Diaconis et Ram, nous avons construit des chaînes de Markov en utilisant une composition du coproduit et produit d'une algébre de Hopf combinatoire. Nous avons présenté un algorithme pour diagonaliser une large classe de ces "chaînes de Hopf puissance", en particulier nous avons diagonalisé le modèle de Gilbert-Shannon-Reeds de mélange de cartes en "riffle shuffle" (couper en deux, puis intercaler) et un modèle de cassage de pierres.

Dans mon travail de thèse, nous supprimons une condition très restrictive de cet article, et ce papier se concentre sur une application de cette amélioration. Nous utilisons ici une nouvelle technique de projection de chaînes de Hopf puissance pour montrer que la chaîne de Hopf puissance sur l'algèbre des fonctions quasi-symétriques est la chaîne de Markov induite sur les ensembles des descentes dans le "riffle shuffling". De plus, nous faisons le lien entre les fonctions propres à droite et à gauche et respectivement les idempotents de Garsia-Reutenauer et les caractères en rubans, ce qui nous permet de retrouver un résultat analogue à Diaconis et Fulman (2012) concernant le nombre de descentes dans le "riffle shuffling".
\end{abstract}

Keywords: Quasisymmetric functions, riffle shuffling, descent set, combinatorial Hopf algebras

\section{Introduction}

The Hopf algebra is a ubiquitous structure in mathematics - having originated in algebraic topology to describe the cohomology of $H$-spaces, it generalises the group ring in representation theory, and is equivalent to a group scheme in algebraic geometry. Joni and Rota(1979) first introduced Hopf algebras to combinatorics to encode the breaking (coproduct) and assembling (product) of combinatorial objects; since then, many examples of the combinatorial Hopf algebra have been developed, for example in Schmitt (1993), and the theory extended in Aguiar and Mahajan (2010).

It is natural to wonder what happens to a combinatorial object after many iterates of breaking and reassembling. Diaconis et al. (2012) examined this question by building a Markov chain out of the 
coproduct-then-product operator on the corresponding combinatorial Hopf algebra. Their main examples of these Hopf-power chains were inverse shuffling (from the free associative algebra, with states indexed by its usual word basis) and rock-breaking (from the algebra of symmetric functions, with states indexed by the elementary symmetric functions $\left\{e_{\lambda}\right\}$ ). The advantage of reformulating these familiar Markov chains as Hopf-power chains is to leverage from theorems concerning arbitrary Hopf algebras. In particular, the Eulerian idempotent theory of Patras (1993) and Reutenauer (1986), which holds for any commutative or cocommutative graded Hopf algebra (over a field of characteristic zero), allows the explicit construction of a left and right eigenbasis of the chain in two rather restrictive circumstances: when the states of the chain form a polynomial basis, or when the underlying Hopf algebra is cocommutative and the states form a free basis. These expressions can aid in estimating convergence rates and probabilities of being in certain subsets of the state space - see (Diaconis et al. 2012, Cor. 4.10, Sec. 2.1) respectively for an example and an extensive list of applications.

The thesis of Pang (in preparation) greatly relaxes the condition that the basis of states be polynomial or free, instead requiring simply that no state of degree greater than 1 is primitive. Thus this extension can, for instance, construct and analyse Markov chains on parking functions and binary trees via the Hopf algebras of Hivert et al. (2008), or use the bases with parameters of Lascoux et al. (2011) to deform familiar chains. The example in the present paper has as its states the non-polynomial basis $\left\{F_{I}\right\}$ of fundamental quasisymmetric functions. The new idea of interpreting Hopf algebra morphisms as a lumping of the corresponding Hopf-power chains shows that (Theorem 3.2) this chain on compositions is the induced chain on descent sets under riffle-shuffling. This descent set chain was briefly studied by (Diaconis and Fulman 2009. Th. 3.2), who gave an upper bound of $\log n$ for the mixing time ( $n$ is the number of cards in the deck). Their emphasis was on the induced chain on the number of descents under riffle-shuffling, for which they proved a mixing time of $\frac{1}{2} \log n$.

Extending the ideas of (Diaconis et al., 2012, Sec. 3.5) yields an explicit algorithm for a full right eigenbasis $\left\{f_{I}\right\}$ and left eigenbasis $\left\{g_{I}\right\}$ of this descent set chain, both indexed by compositions of $n$, where $n$ is the number of cards in the deck. This paper will concentrate on the $f_{I}, g_{I}$ when the parts of $I$ are ordered non-increasing, for which the unwieldly general formula simplifies neatly. This subset of "partition eigenfunctions" completely determine the behaviour of the chain if the starting deck has all cards in increasing order, and they have some surprising interpretations:

- (Theorem 4.2) $f_{I}(J)$ is the coefficient of any permutation with descent set $J$ in the Garsia-Reutenauer idempotent (of the descent algebra) corresponding to $I$;

- (Theorem 4.8 $g_{I}(J)$ is the value of the ribbon character (of the symmetric group) corresponding to $J$ on any permutation of cycle type $I$.

Summing these over partitions of fixed length then recovers the analogous discoveries of Diaconis and Fulman (2012) regarding the Markov chain of the number of descents under riffle-shuffling, see Corollaries 4.7 and 4.12

We remark that the idea of using quasisymmetric functions to analyse descents under riffle-shuffling is not new: Novelli and Thibon (2012) exploited the dual algebra Sym, of noncommutative symmetric functions, to streamline the results of Diaconis and Fulman (2012); (Hersh and Hsiao, 2009, Sec. 7) specialises their theory of walks on quasisymmetric functions to diagonalise the induced chain of riffleshuffling on the idescent set $\{i \mid i+1$ occurs earlier than $i\}$. (They phrase their chain as the descent set under left-multiplication of a certain quasisymmetric function whose right-multiplication describes riffleshuffling; associating the permutation $\sigma$ with the word $\sigma^{-1}(1) \ldots \sigma^{-1}(n)$ instead of $\sigma(1) \ldots \sigma(n)$ then 
exchanges left and right and interprets their chain as the idescent set under shuffling. This results in a genuinely different chain from the one examined in this paper, see the remark in Section 4.1. The remark in (Zhao, 2009. Sec. 2.2) gives further details on the two conventions to notate decks of cards as permutations; to avoid this confusion, this paper will use words instead of permutations.)

This paper is organised as follows. Section 2 collects together the notation necessary to describe the eigenbasis. Section 3 shows that the Hopf-power chain on quasisymmetric functions is the induced chain on descent sets under riffle-shuffling of distinct cards. Section 4 is devoted to the eigenfunctions.

\section{Notation regarding compositions}

A composition $I$ is a list of positive integers $\left(i_{1}, i_{2}, \ldots, i_{l(I)}\right)$. Each $i_{j}$ is a part of $I$. The sum $i_{1}+$ $\cdots+i_{l(I)}$ is denoted $|I|$, and $l(I)$ is the number of parts in $I$. So $|(3,5,2,1)|=11, l((3,5,2,1))=4$. Forgetting the ordering of the parts of $I$ gives a multiset $\lambda(I):=\left\{i_{1}, \ldots, i_{l(I)}\right\}$. Clearly $\lambda(I)=\lambda\left(I^{\prime}\right)$ if and only if $I^{\prime}$ has the same parts as $I$, but in a different order. $I$ is a partition if its parts are non-increasing, that is, $i_{1} \geq i_{2} \geq \cdots \geq i_{l(I)}$.

The diagram of $I$ is a string of $|I|$ dots with a division after the first $i_{1}$ dots, another division after the next $i_{2}$ dots, etc.. The ribbon shape of $I$ is a skew-shape (in the sense of tableaux) with $i_{1}$ boxes in the bottom row, $i_{2}$ boxes in the second-to-bottom row, etc., so that the rightmost square of each row is directly below the leftmost square of the row above. Hence this skew shape contains no 2-by-2 square. The diagram and ribbon shape of $(3,5,2,1)$ are shown below.

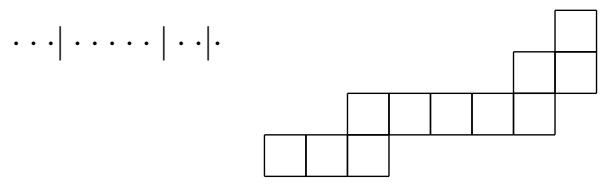

Given compositions $I, J$ with $|I|=|J|$, (Gelfand et al., 1995, Sec. 4.8) defines the decomposition of $J$ relative to $I$ as the $l(I)$-tuple of compositions $\left(J_{1}^{I}, \ldots, J_{l(I)}^{I}\right)$ such that $\left|J_{r}^{I}\right|=i_{r}$ and each $l\left(J_{r}^{I}\right)$ is minimal such that the concatenation $J_{1}^{I} \ldots J_{l(I)}^{I}$ refines $J$. Pictorially, the diagrams of $J_{1}^{I}, \ldots, J_{l(I)}^{I}$ are obtained by "splitting" the diagram of $J$ at the points specified by the divisions in the diagram of $I$. For example, if $I=(4,4,3)$ and $J=(3,5,2,1)$, then $J_{1}^{I}=(3,1), J_{2}^{I}=(4), J_{3}^{I}=(2,1)$.

A composition $I$ is Lyndon if the word $i_{1} \ldots i_{l(I)}$ is lexicographically strictly smaller than its cyclic rearrangements. For example, $(1,1,2,1,2)$ is Lyndon, but $(2,3,2,3)$ and $(3,5,2,1)$ are not. As described by (Lothaire, 1997, Th. 5.1.5, Prop. 5.1.6), the Lyndon factorisation $I_{(1)} \ldots I_{(k)}$ of $I$ is obtained by taking $I_{(k)}$ to be the lexicographically smallest tail of $I$, then $I_{(k-1)}$ is the lexicographically smallest tail of $I$ with $I_{(k)}$ removed, and so on. Hence, if $I=(3,5,2,1)$, then $k(I)=3$ since the Lyndon factors are $I_{(1)}=(3,5), I_{(2)}=(2), I_{(3)}=(1)$. The factors $I_{(r)}$ are important in the general formulae for the full eigenbasis, but this paper will only involve $k(I)$, the number of Lyndon factors in $I$. If $I$ is a partition, then each part of $I$ is a singleton Lyndon factor, which is why the corresponding eigenfunctions have much simpler expressions. In this case, $k(I)=l(I)$.

\section{The Markov chain on descent sets under shuffling}

The purpose of this section is to prove that the coproduct-then-product operator on the algebra of quasisymmetric functions encodes the changes in descent set of a deck of distinct cards under riffle-shuffling. 
Sections 3.1 and 3.2 review background on the shuffle algebra and the algebra of quasisymmetric functions respectively. Section 3.3 defines the all-important Hopf morphism from the shuffle algebra to QSym to relate the two Hopf-power chains, and explains how to lump other Hopf-power chains by the same reasoning. This allows the ideas of (Diaconis et al., 2012. Sec. 3.5) to give explicit expressions for the eigenbasis, as shown in Section 4

\subsection{The Shuffle algebra and riffle-shuffling}

The shuffle algebra $\mathcal{S}$, as defined by $\operatorname{Ree}(1958)$, is spanned by words of the form $w=w_{1} \ldots w_{n}$, where each $w_{i} \in \mathbb{N}$. The $w_{i}$ need not be distinct. $\mathcal{S}$ is multigraded: $\operatorname{deg}(w)=\left(\left|\left\{i: w_{i}=1\right\}\right|,\left|\left\{i: w_{i}=2\right\}\right|, \ldots\right)$. In other words, the $k$ th component of $\operatorname{deg}(w)$ is the number of times the letter $k$ appears in $w$. The shuffle algebra also admits a coarser grading: $|w|$ is the number of letters in w. For example, $\operatorname{deg}(12231)=$ $(2,2,1),|12231|=5$.

The product of two words $w$ and $w^{\prime}$, denoted $m\left(w \otimes w^{\prime}\right)$, is the sum of all possible interleavings of their letters, with multiplicity. For example,

$$
\begin{gathered}
m(13 \otimes 52)=1352+1532+1523+5132+5123+5213 ; \\
m(12 \otimes 231)=2(12231)+12321+12312+21231+21321+21312+2(23112)+23121 .
\end{gathered}
$$

Iterating this gives the $a$-fold product:

$$
m^{[a]}: \mathcal{S}^{\otimes a} \rightarrow \mathcal{S}, \quad m^{[1]}:=\iota, \quad m^{[a]}:=m\left(m^{[a-1]} \otimes \iota\right),
$$

where $\iota$ denotes the identity map. Note that $m=m^{[2]}$. (Reutenauer, 1993, Sec. 1.5) showed that deconcatenation is a compatible coproduct. For example,

$$
\Delta(316)=\emptyset \otimes 316+3 \otimes 16+31 \otimes 6+316 \otimes \emptyset .
$$

(Here, $\emptyset$ denotes the empty word, which is the unit of $\mathcal{S}$.) The $a$-fold coproduct is given inductively by:

$$
\Delta^{[a]}: \mathcal{S} \rightarrow \mathcal{S}^{\otimes a}, \quad \Delta^{[1]}:=\iota, \quad \Delta^{[a]}:=\left(\Delta^{[a-1]} \otimes \iota\right) \Delta,
$$

so again $\Delta=\Delta^{[2]}$. As an example,

$$
\begin{aligned}
& \Delta^{[3]}(316)=\emptyset \otimes \emptyset \otimes 316+\emptyset \otimes 3 \otimes 16+3 \otimes \emptyset \otimes 16+\emptyset \otimes 31 \otimes 6+3 \otimes 1 \otimes 6 . \\
&+31 \otimes \emptyset \otimes 6+\emptyset \otimes 316 \otimes \emptyset+3 \otimes 16 \otimes \emptyset+31 \otimes 6 \otimes \emptyset+316 \otimes \emptyset \otimes \emptyset
\end{aligned}
$$

Letting the word $w_{1} \ldots w_{n}$ represent a deck of cards in the order $w_{1}, w_{2}, \ldots, w_{n}$ from top to bottom, the Hopf-square map $\Psi^{2}:=m \Delta$ represents a Gilbert-Shannon-Reeds shuffle: cut the deck binomially with parameter $\frac{1}{2}$, then drop the cards one by one from either pile, where the chance of dropping from a pile is proportional to the number of cards currently in the pile. Precisely,

$$
\Psi^{2}(w)=m \Delta(w)=\sum_{w^{\prime}} 2^{|w|} K_{2}\left(w, w^{\prime}\right) w^{\prime}
$$

where $K_{2}\left(w, w^{\prime}\right)$ is the chance of a GSR shuffle applied to $w$ resulting in $w^{\prime}$. In other words, the matrix for the operator $2^{-n} \Psi^{2}$, with respect to the basis of words with $n$ letters, transposes to give the transition 
matrix of the GSR shuffle. Analogously, the ath Hopf-power, $\Psi^{a}:=m^{[a]} \Delta^{[a]}$, describes the $a$-shuffle of Bayer and Diaconis (1992), where the cards are cut into $a$ piles multinomially (with parameter $\frac{1}{a}$ ) and then dropped proportional to pile size as before.

The descent set of a word $w=w_{1} \ldots w_{n}$ is defined to be $D(w)=\left\{j \in\{1,2, \ldots,|w|-1\} \mid w_{j}>w_{j+1}\right\}$. It is more convenient in this paper to consider the associated composition of $D(w)$. Hence a word $w$ has descent composition $D C(w)=I$ if $i_{j}$ is the number of letters between the $j-1$ th and $j$ th descent, i.e. if $w_{i_{1}+\cdots+i_{j}}>w_{i_{1}+\cdots+i_{j}+1}$ for all $j$, and $w_{r} \leq w_{r+1}$ for all $r \neq i_{1}+\cdots+i_{j}$. For example, $D(3521)=\{2,3\}$ and $D C(3521)=(2,1,1)$. Note that no information is lost in passing from $D(w)$ to $D C(w)$, as the divisions in the diagram of $D C(w)$ indicate the positions of descents in $w$.

\subsection{The algebra of quasisymmetric functions}

The algebra QSym of quasisymmetric functions was first introduced by Gessel (1984) to study $P$ partitions. It is a subalgebra of the algebra of polynomials in infinitely many commuting variables $\left\{x_{1}, x_{2}, \ldots\right\}$. Gessel defined two bases of $Q S y m$, both indexed by compositions. The monomial quasisymmetric function $M_{I}$ associated to a composition $I=\left(i_{1}, \ldots, i_{l(I)}\right)$ is

$$
M_{I}=\sum_{j_{1}<\cdots<j_{l(I)}} x_{j_{1}}^{i_{1}} \ldots x_{j_{l(I)}}^{i_{l(I)}},
$$

and the fundamental quasisymmetric function $F_{I}$ associated to $I$ is

$$
F_{I}=\sum_{J \geq I} M_{J}
$$

where the sum runs over all partitions $J$ refining $I$. QSym inherits a grading and a commutative product from the algebra of polynomials, so $\operatorname{deg}\left(M_{I}\right)=\operatorname{deg}\left(F_{I}\right)=|I|$. Malvenuto and Reutenauer (1995) extended this to a Hopf algebra structure by defining the following coproduct:

$$
\Delta\left(M_{I}\right)=\sum_{j=0}^{l(I)} M_{\left(i_{1}, i_{2}, \ldots, i_{j}\right)} \otimes M_{\left(i_{j+1}, \ldots, i_{l(I)}\right)} .
$$

Equations (1) and (2) define an $a$-fold product and $a$-fold coproduct on QSym.

\subsection{Lumping riffle-shuffling by descent set}

The algebraic relationship between the two Hopf algebras above is:

Theorem 3.1 There is a morphism of Hopf algebras $\theta: \mathcal{S} \rightarrow$ QSym such that, if $w$ is a word with distinct letters, then $\theta(w)=F_{D C(w)}$.

Proof (sketch): The linear function $\zeta: \mathcal{S} \rightarrow \mathbb{R}$ defined by

$$
\zeta(w)= \begin{cases}1 & \text { if } w_{1}<w_{2}<\cdots<w_{n} \\ 0 & \text { otherwise }\end{cases}
$$

is an algebra homomorphism; now apply the universal construction of (Aguiar et al., 2006, Th. 4.1).

As $\theta$ is a Hopf morphism, it commutes with the $a$ th Hopf-power map $\Psi^{a}$. The probability interpretation of this is: 
Theorem 3.2 The descent set process of a deck of $n$ distinct cards under a-shuffling is a Markov chain, whose transition matrix $\bar{K}_{a, n}$ is the transpose of the matrix for the rescaled Hopf-power map $a^{-n} \Psi^{a}$ on QSym, with respect to the basis $\left\{F_{I}|| I \mid=n\right\}$.

Proof: Let $K_{a, n}$ be the transition matrix for $a$-shuffling on a deck of $n$ distinct cards. Then, for any $w$ with $n$ distinct letters,

$$
a^{-n} \Psi^{a}(w)=\sum_{w^{\prime}} K_{a, n}\left(w, w^{\prime}\right) w^{\prime}
$$

Apply $\theta$ of Theorem 3.1 to both sides, remembering that $\theta$ and $\Psi^{a}$ commute:

$$
a^{-n} \Psi^{a}(\theta(w))=\sum_{w^{\prime}} K_{a, n}\left(w, w^{\prime}\right) \theta\left(w^{\prime}\right) .
$$

As all words involved have distinct letters, Theorem 3.1 yields

$$
a^{-n} \Psi^{a}\left(F_{D C(w)}\right)=\sum_{w^{\prime}} K_{a, n}\left(w, w^{\prime}\right) F_{D C\left(w^{\prime}\right)}=\sum_{J}\left(\sum_{w^{\prime}: D C\left(w^{\prime}\right)=J} K_{a, n}\left(w, w^{\prime}\right)\right) F_{J} .
$$

The left hand side of this equation depends only on $D C(w)$, so the same is true of the coefficients $\sum_{w^{\prime}: D C\left(w^{\prime}\right)=J} K_{a, n}\left(w, w^{\prime}\right)$ on the right. These are the probabilities that, after an $a$-shuffle, a deck in order $w$ now has descent composition $J$. Hence the descent set process under shuffling is indeed a Markov chain, and Equation 3 gives the transition probabilities as the $(I, J)$-entry of the transpose of the matrix for $a^{-n} \Psi^{a}$.

Remark. This straightforward argument applies verbatim to lump Hopf-power Markov chains on other graded Hopf algebras. Let $\theta: \mathcal{H} \rightarrow \mathcal{H}^{\prime}$ be a morphism of graded Hopf algebras mapping a basis $\mathcal{B}_{\nu}$ of the degree $\nu$ subspace $\mathcal{H}_{\nu}$ of $\mathcal{H}$ onto a basis $\mathcal{B}_{\nu^{\prime}}^{\prime}$ of some degree $\nu^{\prime}$ subspace $\mathcal{H}_{\nu^{\prime}}^{\prime}$ of $\mathcal{H}^{\prime} .\left(\theta: \mathcal{H}_{\nu} \rightarrow \mathcal{H}_{\nu^{\prime}}^{\prime}\right.$ must be surjective, but need not be injective - several elements of $\mathcal{B}_{\nu}$ may have the same image in $\mathcal{H}_{\nu^{\prime}}^{\prime}$, as long as the distinct images are linearly independent.) Then the Hopf-power walk on $\mathcal{B}_{\nu}$ lumps via $\theta$ to the Hopf-power walk on $\mathcal{B}_{\nu^{\prime}}^{\prime}$.

\section{Explicit formulae for eigenfunctions}

Section 4.1 gives the eigenvalues of the descent set chain and their multiplicities. Sections 4.2 and 4.3 detail formulae for the right and left "partition eigenfunctions" respectively, explain how to recover the results of Diaconis and Fulman (2012), and sketch the ideas behind the proofs of the full eigenbases. This strategy also diagonalises Hopf-power chains on a large class of commutative cofree combinatorial Hopf algebras. These eigenfunctions are useful for a variety of probabilistic tasks, as (Diaconis et al. 2012. Sec. 2.1) explains.

\subsection{Multiplicity of eigenvalues}

As is the case for previously analysed Hopf-power Markov chains, all eigenvalues of this descent set chain are powers of the Hopf-power exponent $a$. The full eigenbasis algorithm shows that $f_{I}$ has eigenvalue $a^{k(I)-|I|}$, where $k(I)$ is the number of Lyndon factors in $I$. A standard generating function argument then rephrases this as: 
Theorem 4.1 The eigenvalues of the ath Hopf-power Markov chain on compositions of $n$ are $1, a^{-1}, a^{-2}, \ldots, a^{-n+1}$. The multiplicity of the eigenvalue $a^{-n+k}$ is the coefficient of $x^{n} y^{k}$ in $\prod_{i}\left(1-y x^{i}\right)^{-d_{i}}$, where $d_{i}$ is the number of Lyndon compositions I with $|I|=i$.

Remark. The idescent set chain of (Hersh and Hsiao, 2009. Sec. 7) has the same eigenvalues, but there each composition $I$ corresponds to an eigenfunction of eigenvalue $a^{-n+l(I)}$, so the multiplicity of $a^{-n+k}$ in the idescent set chain is the number of compositions of $n$ with length $k$. This difference in eigenvalue multiplicity suggests the two chains have different convergence rates.

\subsection{Right eigenfunctions}

All functions $f_{I}$ in the right eigenbasis are essentially built from the function

$$
f(J):=\frac{1}{|J|} \frac{(-1)^{l(J)-1}}{\left(\begin{array}{c}
|J|-1 \\
l(J)-1
\end{array}\right)} .
$$

Note that $f(J)$ depends only on $|J|$ and $l(J)-1$, which are respectively the number of dots and the number of divisions in the diagram of $J$.

Theorem 4.2 below gives an explicit formula for the right eigenfunctions $f_{I}$ corresponding to a partition $I$ (when $I$ is not a partition, the sum becomes weighted), and relates them to the orthogonal idempotents $E_{I}$ of the descent algebra. These idempotents refine the more familiar Eulerian idempotent, and were first defined by (Garsia and Reutenauer, 1989. Sec. 3) to classify indecomposable representations of the descent algebra.

Theorem 4.2 Let I be a partition with $|I|=n$. With $f$ as defined above, the function

$$
f_{I}(J):=\frac{1}{l(I) !} \sum_{I^{\prime}: \lambda\left(I^{\prime}\right)=\lambda(I)} \prod_{r=1}^{l\left(I^{\prime}\right)} f\left(J_{r}^{I^{\prime}}\right)=\frac{1}{l(I) ! i_{1} \ldots i_{l(I)}} \sum_{I^{\prime}: \lambda\left(I^{\prime}\right)=\lambda(I)} \prod_{r=1}^{l\left(I^{\prime}\right)} \frac{(-1)^{l\left(J_{r}^{I^{\prime}}\right)-1}}{\left(\begin{array}{l}
\left|J_{r}^{I^{\prime}}\right|-1 \\
l\left(J_{r}^{I^{\prime}}\right)-1
\end{array}\right)}
$$

is a right eigenfunction of eigenvalue $a^{-n+l(I)}$ of the ath Hopf-power Markov chain on compositions. The numbers $f_{I}(J)$ are the coefficients in the Garsia-Reutenauer idempotent $E_{I}$ :

$$
E_{I}=\sum_{\sigma \in \mathfrak{S}_{n}} f_{I}(D C(\sigma)) \sigma .
$$

Observe that $f$ itself is a right eigenfunction, that corresponding to the partition with single part. Its eigenvalue is $a^{-n+1}$, the smallest possible.

Example 4.3 Here's how to apply the algorithm above to calculate $f_{(4,4,3)}((3,5,2,1))$. The $I^{\prime}$ in the sum are the rearrangements of $(4,4,3)$, which are $(4,4,3),(4,3,4)$ and $(3,4,4)$. The decompositions of $(3,5,2,1)$ with respect to these three $I^{\prime}$ are:

$$
(\cdots|\cdot, \cdots, \cdot \cdot| \cdot) \quad(\cdots|\cdot, \cdots, \cdot| \cdot \mid \cdot) \quad(\cdots, \cdots, \cdot|\cdot| \cdot)
$$

so

$$
f_{(4,4,3)}((3,5,2,1))=\frac{1}{3 !}\left(\frac{-1}{4\left(\begin{array}{l}
3 \\
1
\end{array}\right)} \frac{1}{4} \frac{-1}{3\left(\begin{array}{l}
2 \\
1
\end{array}\right)}+\frac{-1}{4\left(\begin{array}{l}
3 \\
1
\end{array}\right)} \frac{1}{3} \frac{1}{4\left(\begin{array}{c}
3 \\
2
\end{array}\right)}+\frac{1}{3} \frac{1}{4} \frac{1}{4\left(\begin{array}{c}
3 \\
2
\end{array}\right)}\right)=\frac{7}{5184}
$$


As $f((1))=1$, one may omit all $r$ with $\left|J_{r}^{I^{\prime}}\right|=1$ from the product in the expression for $f_{I}$. This simplifies the calculation of $f_{\left(i_{1}, 1,1, \ldots, 1\right)}(J)$ to "pulling a window" of length $i_{1}$ across the diagram of $J$ and summing the values of $f$ on each position of the window.

Example 4.4 Take $i_{1}=2$, then in the window of length 2, there is either a division or no division. Since $f((2))=\frac{1}{2}$ and $f((1,1))=-\frac{1}{2}, f_{(2,1,1, \ldots, 1)}(J)$ is the sum of $\frac{1}{2}$ for every non-division and $-\frac{1}{2}$ for every division, divided by $(n-1)$ !, i.e.,

$$
f_{(2,1,1, \ldots, 1)}(J)=\frac{1}{(n-1) !}\left(\frac{|J|-1}{2}-(l(J)-1)\right) .
$$

By Theorem 4.1. this is the unique right eigenfunction of eigenvalue $\frac{1}{a}$, the largest eigenvalue after 1. Its lift to the shuffle algebra is the "normalised number of descents" eigenfunction, as discussed in (Diaconis et al. 2012, Ex. 5.8).

Example 4.5 When $i_{1}=3$, calculations of $f(J)$ for $J$ with $|J|=3$ show that

$$
\begin{aligned}
f_{(3,1,1, \ldots, 1)}(J)= & \frac{1}{3(n-2) !}(\#(2 \text { consecutive non-divisions })+\#(2 \text { consecutive divisions }) \\
& \left.\left.-\frac{1}{2} \#(\text { division followed by non-division })-\frac{1}{2} \# \text { (non-division followed by division }\right)\right) .
\end{aligned}
$$

The associated eigenvalue is $\frac{1}{a^{2}}$. Since divisions correspond to descents in the shuffle algebra, and nondivisions to ascents, $f_{(3,1,1, \ldots, 1)}$ lifts to the shuffle algebra as

$$
\begin{aligned}
\tilde{f}_{(3,1,1, \ldots, 1)}(w) & =\frac{1}{3(n-2) !}\left(\# \text { straights }-\frac{1}{2} \# \text { troughs }-\frac{1}{2} \# \text { peaks }\right) \\
& =\frac{1}{2(n-2) !}\left(\# \text { straights }-\frac{n-2}{3}\right)
\end{aligned}
$$

since \# straights $+\#$ troughs $+\#$ peaks $=n-2$. (Here, a straight is two consecutive ascents or two consecutive descents.) This "normalised number of straights" eigenfunction is $\frac{1}{2(n-2) !} f_{-}$in the notation of (Diaconis et al. 2012, Prop.5.10). The full eigenbasis formula shows that the normalised number of peaks and of troughs are also eigenfunctions. Consequently:

Proposition 4.6 The expected number of straights (resp. peaks, troughs) after l shuffles, starting from a deck with $x$ straights (resp. peaks, troughs), is

$$
\left(1-4^{-l}\right) \frac{n-2}{3}+4^{-l} x
$$

The story is similar for larger $i_{1}: f_{\left(i_{1}, 1,1, \ldots, 1\right)}$ is the weighted enumeration of "patterns" of length $i_{1}$, where pattern $J$ has weight $\frac{f(J)}{\left(n-i_{1}+1\right) !}$. Each of these lifts to an eigenfunction on the shuffle algebra, that is a weighted enumeration of up-down-patterns of length $i_{1}$.

Corollary 4.7 (Diaconis and Fulman 2012, Cor. 3.2) Let $f_{i}(j)$ be the coefficient of any permutation with $j$ descents in the ith Eulerian idempoten. Then $\left\{f_{i}\right\}$ is a right eigenbasis for the Markov chain on the number of descents under riffle-shuffling. 
Proof: The key is that the Garsia-Reutenauer idempotents for partitions of a fixed length sum to the corresponding Eulerian idempotent:

$$
e_{i}=\sum_{l(I)=i} E_{I}=\sum_{\sigma \in \mathfrak{S}_{n}} \sum_{l(I)=i} f_{I}(D C(\sigma)) \sigma
$$

Since the coefficient of a permutation in the Eulerian idempotent depends only on its number of descents, the function $\sum_{l(I)=i} f_{I}(J)$ depends only on $l(J)$, and it is a right eigenfunction of eigenvalue $a^{-n+i}$. By the eigenfunction theory of lumped chains, as in (Levin et al., 2009, Lem. 12.8.i), this descends to a right eigenfunction

$$
j \rightarrow \sum_{l(I)=i} f_{I}(J) \text { for any } J \text { of length } j
$$

on the induced chain on the number of descents, which is the required $f_{i}$.

The full right eigenbasis $\left\{f_{I}\right\}$ comes from applying (Diaconis et al., 2012, Th. 3.16) to Sym, the graded dual of $Q S y m$. The eigenfunctions are most naturally expressed in terms of $\left\{\Phi^{I}\right\}$, the noncommutative power sum of the second kind; one then uses the explicit change-of-basis matrices of (Gelfand et al. 1995, Sec. 4) to rewrite this in terms of the dual basis to $\left\{F_{I}\right\}$, which is $\left\{R_{I}\right\}$, the noncommutative ribbon symmetric functions.

\subsection{Left eigenfunctions}

The left eigenfunctions $g_{I}$ for $I$ a partition are most concisely defined using some representation theory of the symmetric group $\mathfrak{S}_{n}$, although their calculation is completely combinatorial. Each composition $J$ may be associated to a representation of $\mathfrak{S}_{n}$ via its ribbon shape $J$; denote by $\chi^{J}$ the character of this representation.

Theorem 4.8 Let $I$ be a partition with $|I|=n$. Define $g_{I}(J):=\chi^{J}(I)$, the character of $\mathfrak{S}_{n}$ associated to the ribbon shape $J$ evaluated at a permutation with cycle type $I$. Then $g_{I}$ is a left eigenfunction of the ath Hopf-power Markov chain on compositions with eigenvalue $a^{-n+l(I)}$.

(Ceccherini-Silberstein et al. 2010, Rem. 3.5.18) explains how to calculate $\chi^{J}(I)$ graphically: find all possible ways of filling the ribbon shape of $J$ with $i_{1}$ copies of $1, i_{2}$ copies of 2 , etc., such that all copies of each integer are in adjacent cells, and all rows and columns are weakly increasing; then sum over these fillings, weighted by $(-1)^{\Sigma\left(l_{r}-1\right)}$, where $l_{r}$ is the number of rows containing $r$. (For general compositions $I$, the left eigenfunction $g_{I}(J)$ is a weighted sum over coloured fillings of the ribbon shape of $J$ subject to complex restrictions, and does not have a neat expression in terms of characters.)

Example 4.9 Calculating $g_{(4,4,3)}((3,5,2,1))$ requires filling the ribbon shape of $(3,5,2,1)$ with four copies of 1 , four copies of 2 and three copies of 3 , subject to the constraints in explained above. Observe that the top square cannot be 1 , because then the top four squares must all contain 1 , and the fifth square from the top must be equal to or smaller than these. Similarly, the top square cannot be 3 , because then the top three squares are all 3s, but the fourth must be equal or larger. Hence 2 must fill the top square, and the only legal way to complete this is

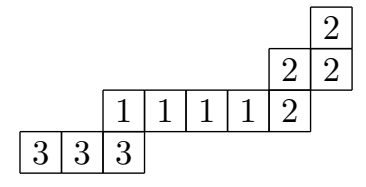


so

$$
g_{(4,4,3)}((3,5,2,1))=(-1)^{(0+2+0)}=1 .
$$

Example 4.10 There is only one way to fill any given ribbon shape with $n$ copies of 1, so

$$
g_{(n)}(J)=(-1)^{l(J)-1} .
$$

Next, take $I=(1,1, \ldots, 1)$. Then $g_{(1,1, \ldots, 1)}$ is $\chi^{J}$ evaluated on the identity permutation. A theorem of Foulkes (1980), described in Kerber and Thürlings (1984), translates this to:

Corollary 4.11 The stationary distribution for the ath Hopf-power Markov chain on compositions is

$$
g_{(1,1, \ldots, 1)}(J)=\frac{1}{n !}|\{w|| w \mid=n, \operatorname{deg}(w)=(1,1, \ldots, 1), D C(w)=J\}|
$$

In other words, the stationary probability of $J$ is the proportion of words with letters are $1,2, \ldots, n$ (each appearing exactly once) whose descent composition is $J$.

This also follows from the stationary distribution of riffle-shuffling being the uniform distribution.

Corollary 4.12 (Diaconis and Fulman 2012 Th. 2.1) Let $g_{i}(j)$ be the value of the jth Foulkes character of the symmetric group on any permutation with $i$ cycles. Then $\left\{g_{i}\right\}$ is a left eigenbasis for the Markov chain on the number of descents under riffle-shuffing.

Proof: Each $g_{I}$ determines a left eigenfunction for the number of descents chain, by summing the values of $g_{I}$ over all compositions lumping to the same state, see (Barr and Thomas, 1977, Th. 2) for details. So

$$
j \rightarrow \sum_{l(J)=j} g_{I}(J)=\sum_{l(J)=j} \chi^{J}(I)
$$

is an eigenfunction of eigenvalue $a^{-n+l(I)}$, and this sum of ribbon characters is by definition the $j$ th Foulkes character.

The full left eigenbasis is given by essentially applying (Diaconis et al., 2012, Th. 3.15) to products of $\left\{P_{I} \mid I\right.$ is Lyndon $\}$, where $\left\{P_{I}\right\}$ is the power sum analogue as defined by (Malvenuto and Reutenauer. 1995. Eq. 2.12), as their Corollary 2.2 states that $\left\{P_{I} \mid I\right.$ is Lyndon $\}$ freely generates $Q S y m$.

\section{Acknowledgements}

I would like to thank my advisor Persi Diaconis for introducing this line of investigation to me and bringing to my attention several of the references. I am grateful to Sami Assaf, Nantel Bergeron, Dan Bump, Dave Hill, Anthony Licata, Jean-Christophe Novelli and Ravi Vakil for helpful conversations. SAGE computer software (Stein et al. (2012)), in particular the noncommutative symmetric functions code by Jason Bandlow, Chris Berg, Franco Saliola and Nicolas M. Thiéry, was extremely useful, thanks to Sam Lichtenstein and Simon Rubenstein-Salzedo for tips with working with SAGE. Thanks also to Megan Bernstein, Isabelle Camilier and Daniel Kim Murphy for assistance in preparing this article, and to the reviewer for his/her detailed constructive feedback. 


\section{References}

M. Aguiar and S. Mahajan. Monoidal functors, species and Hopf algebras, volume 29 of CRM Monograph Series. American Mathematical Society, Providence, RI, 2010. ISBN 978-0-8218-4776-3.

M. Aguiar, N. Bergeron, and F. Sottile. Combinatorial Hopf algebras and generalized Dehn-Sommerville relations. Compos. Math., 142(1):1-30, 2006. ISSN 0010-437X.

D. R. Barr and M. U. Thomas. An eigenvector condition for Markov chain lumpability. Operations Res., 25(6):1028-1031, 1977. ISSN 0030-364X.

D. Bayer and P. Diaconis. Trailing the dovetail shuffle to its lair. Ann. Appl. Probab., 2(2):294-313, 1992. ISSN 1050-5164.

T. Ceccherini-Silberstein, F. Scarabotti, and F. Tolli. Representation theory of the symmetric groups, volume 121 of Cambridge Studies in Advanced Mathematics. Cambridge University Press, Cambridge, 2010. ISBN 978-0-521-11817-0.

P. Diaconis and J. Fulman. Carries, shuffling, and symmetric functions. Adv. in Appl. Math., 43(2): 176-196, 2009. ISSN 0196-8858.

P. Diaconis and J. Fulman. Foulkes characters, Eulerian idempotents, and an amazing matrix. J. Algebraic Combin., 36(3):425-440, 2012. ISSN 0925-9899. doi: 10.1007/s10801-012-0343-7. URL http: //dx.doi.org/10.1007/s10801-012-0343-7.

P. Diaconis, C. Y. A. Pang, and A. Ram. Hopf algebras and Markov chains: two examples and a theory. ArXiv e-prints, June 2012.

H. O. Foulkes. Eulerian numbers, Newcomb's problem and representations of symmetric groups. Discrete Math., 30(1):3-49, 1980. ISSN 0012-365X. doi: 10.1016/0012-365X(80)90061-8. URL http: //dx.doi.org/10.1016/0012-365x(80)90061-8.

A. M. Garsia and C. Reutenauer. A decomposition of Solomon's descent algebra. Adv. Math., 77(2): 189-262, 1989. ISSN 0001-8708.

I. M. Gelfand, D. Krob, A. Lascoux, B. Leclerc, V. S. Retakh, and J.-Y. Thibon. Noncommutative symmetric functions. Adv. Math., 112(2):218-348, 1995. ISSN 0001-8708. doi: 10.1006/aima.1995.1032. URL http://dx.doi.org/10.1006/aima.1995.1032.

I. M. Gessel. Multipartite $P$-partitions and inner products of skew Schur functions. In Combinatorics and algebra (Boulder, Colo., 1983), volume 34 of Contemp. Math., pages 289-317. Amer. Math. Soc., Providence, RI, 1984. doi: 10.1090/conm/034/777705. URL http://dx.doi.org/10.1090/ conm/034/777705.

P. Hersh and S. K. Hsiao. Random walks on quasisymmetric functions. Adv. Math., 222(3):782-808, 2009. ISSN 0001-8708. doi: 10.1016/j.aim.2009.05.014. URL/http://dx.doi.org/10.1016/ j.aim.2009.05.014. 
F. Hivert, J.-C. Novelli, and J.-Y. Thibon. Commutative combinatorial Hopf algebras. J. Algebraic Combin., 28(1):65-95, 2008. ISSN 0925-9899. doi: 10.1007/s10801-007-0077-0. URL http: //dx.doi.org/10.1007/s10801-007-0077-0.

S. A. Joni and G.-C. Rota. Coalgebras and bialgebras in combinatorics. Stud. Appl. Math., 61(2):93-139, 1979. ISSN 0022-2526.

A. Kerber and K.-J. Thürlings. Eulerian numbers, foulkes characters and lefschetz characters of $S_{n}, 1984$.

A. Lascoux, J.-C. Novelli, and J.-Y. Thibon. Noncommutative symmetric functions with matrix parameters. ArXiv e-prints, Oct. 2011.

D. A. Levin, Y. Peres, and E. L. Wilmer. Markov chains and mixing times. American Mathematical Society, Providence, RI, 2009. ISBN 978-0-8218-4739-8.

M. Lothaire. Combinatorics on Words. Cambridge Mathematical Library. Cambridge University Press, Cambridge, 1997. ISBN 0-521-59924-5.

C. Malvenuto and C. Reutenauer. Duality between quasi-symmetric functions and the Solomon descent algebra. J. Algebra, 177(3):967-982, 1995. ISSN 0021-8693. doi: 10.1006/jabr.1995.1336. URL http://dx.doi.org/10.1006/jabr.1995.1336

J.-C. Novelli and J.-Y. Thibon. Noncommutative symmetric functions and an amazing matrix. Adv. in Appl. Math., 48(3):528-534, 2012. ISSN 0196-8858. doi: 10.1016/j.aam.2011.11.008. URL http: //dx.doi.org/10.1016/j.aam.2011.11.008.

C. Y. A. Pang. Hopf algebras and markov chains. in preparation.

F. Patras. La décomposition en poids des algèbres de Hopf. Ann. Inst. Fourier (Grenoble), 43(4):10671087, 1993. ISSN 0373-0956.

R. Ree. Lie elements and an algebra associated with shuffles. Ann. of Math. (2), 68:210-220, 1958. ISSN 0003-486X.

C. Reutenauer. Theorem of Poincaré-Birkhoff-Witt, logarithm and symmetric group representations of degrees equal to Stirling numbers. In Combinatoire énumérative (Montreal, Que., 1985/Quebec, Que., 1985), volume 1234 of Lecture Notes in Math., pages 267-284. Springer, Berlin, 1986. doi: 10.1007/ BFb0072520. URL http://dx.doi.org/10.1007/BFb0072520.

C. Reutenauer. Free Lie Algebras, volume 7 of London Mathematical Society Monographs. New Series. The Clarendon Press Oxford University Press, New York, 1993. ISBN 0-19-853679-8. Oxford Science Publications.

W. R. Schmitt. Hopf algebras of combinatorial structures. Canad. J. Math., 45(2):412-428, 1993. ISSN 0008-414X.

W. Stein et al. Sage Mathematics Software (Version 5.4.1). The Sage Development Team, 2012. http: //www.sagemath.org.

Y. Zhao. Biased riffle shuffles, quasisymmetric functions, and the RSK algorithm. 2009. URL http: //yufeizhao.com/papers/shuffling.pdf 